\title{
Serum uric acid in HIV infected children and correlation to cardiovascular risk factors
}

\author{
Aleksandra Stańska-Perka ${ }^{1}$, Magdalena Marczyńska ${ }^{1,2}$, Konrad Zawadka $^{1,2}$, Jolanta Popielska ${ }^{1,2}$ \\ ${ }^{1}$ Hospital of Infectious Diseases, Warsaw, Poland \\ ${ }^{2}$ Department of Children's Infectious Diseases, Medical University of Warsaw, Poland
}

\begin{abstract}
Introduction: Serum uric acid (SUA) has been reported to be associated with hypertension and metabolic syndrome. Metabolic disorders leading to increased cardiovascular risk are often observed in human immunodeficiency virus (HIV)-infected children. The aim of the study was to assess the level of SUA and its association with metabolic abnormalities and high blood pressure.
\end{abstract}

Material and methods: Fasting SUA, total cholesterol, triglyceride, high-density lipoprotein cholesterol (HDL-C), low-density lipoprotein cholesterol (LDL-C), insulin, and glucose measurements were performed in $54 \mathrm{HIV}$-infected children (mean age 12.3 years), $46 \%$ male. All but two were treated with highly active antiretroviral therapy (HAART). HOMA-IR (Homeostatic Model Assessment of Insulin Resistance) was calculated using the formula HOMA-IR = (insulin [mU/l] $\times$ glucose $[\mathrm{mmol} / \mathrm{l}]) / 22.5$. All children underwent anthropometric measurements. Body mass index (BMI) was calculated by dividing weight in kilograms by square of height in metres.

Results: Mean SUA was $239.8 \mu \mathrm{mol} / \mathrm{l}$, and no patient showed hiperuricaemia. SUA was higher in boys and in older children. Nadir immunologic category 3 was associated with higher SUA compared to category 1 and 2 together. Children with abnormal triglycerides, HDL-C, insulin, and HOMA-IR had significantly higher SUA. Regression analysis showed that all metabolic parameters apart from HDL-C influence SUA. Children treated with HAART based on non-nucleoside reverse transcriptase inhibitors (NNRTIs) had lower SUA in contrast to children receiving a protease inhibitor (PI)-based or other HAART scheme. We did not observe any association between SUA and high blood pressure.

Conclusions: We showed correlation between SUA and metabolic parameters in HIV-infected children. Metabolic abnormalities are the result of both HIV infection and antiretroviral treatment. Association of SUA with CD4 nadir and HAART may indicate the effect of HIV itself or its treatment.

HIV AIDS Rev 2019; 18, 1: 19-24 DOI: https://doi.org/10.5114/hivar.2019.84141

Key words: serum uric acid, cardiovascular risk, HAART.

\section{Introduction}

Serum uric acid (SUA) has been reported to be associated with multiple metabolic abnormalities: hypertension, meta- bolic syndrome, cardiovascular disease, and renal failure [1-4]. Its usefulness as a marker of cardiovascular abnormalities is observed especially in high-risk populations $[2,5]$. SUA concentration is higher in obese children compared
Address for correspondence: Dr. Aleksandra Stańska-Perka, Hospital of Infectious Diseases, 37 Wolska St., 01-201 Warsaw, Poland, e-mail: a_stanska@tlen.pl
Article history:

Received: 13.05.2018

Received in revised form: 19.05.2018

Accepted: 06.02.2019

Available online: 28.03.2019
International Journal of HIV-Related Problems

HIV \& AIDS

R e v i e w 
Table 1. General characteristics of the study group

\begin{tabular}{|c|c|}
\hline Age, y mean (SD) & $12.02(4.35)$ \\
\hline Male, $n(\%)$ & $25(46)$ \\
\hline HAART, yes, $n(\%)$ & $52(96)$ \\
\hline \multicolumn{2}{|l|}{ Immunological status, $n$ (\%) } \\
\hline 1 & $50(92.5)$ \\
\hline 2 & $4(7.5)$ \\
\hline 3 & 0 \\
\hline \multicolumn{2}{|l|}{ Clinical status, $n(\%)$} \\
\hline A & $47(87)$ \\
\hline B & $1(2)$ \\
\hline C & $6(11)$ \\
\hline BMI z-score, mean (SD) & $0.01(0.83)$ \\
\hline SBP z-score, mean (SD) & $0.07(1.24)$ \\
\hline DBP z-score, mean (SD) & $0.39(0.88)$ \\
\hline SUA, $\mu \mathrm{mol} / \mathrm{l}$, mean (SD) & $239.8(65.7)$ \\
\hline $\mathrm{TC}, \mathrm{mg} / \mathrm{dl}$, mean (SD) & $179.9(36.7)$ \\
\hline HDL-C, mg/dl, mean (SD) & $60.1(19)$ \\
\hline LDL-C, mg/dl, mean (SD) & $93.7(29.6)$ \\
\hline TG, mg/dl, mean (SD) & $171.4(95.5)$ \\
\hline Insulin, $\mu \mathrm{mol} / \mathrm{ml}$, mean (SD) & $14.2(11.5)$ \\
\hline Fasting glucose, mg/dl, mean (SD) & $87.5(7.1)$ \\
\hline Glucose in $120^{\prime}$ OGTT, mean (SD) & $98.9(23.8)$ \\
\hline HOMA-IR, mean (SD) & $2.86(1.83)$ \\
\hline \multicolumn{2}{|c|}{$\begin{array}{l}\text { HAART - highly active antiretroviral therapy, BMI - body mass index, } \\
\text { SBP - systolic blood pressure, DBP - diastolic blood pressure, SUA - serum } \\
\text { uric acid, TC - total cholesterol, HDL-C - high-density lipoprotein cholesterol, } \\
\text { LDL-C - low-density lipoprotein cholesterol, TG - triglyceride, OGTT - oral } \\
\text { glucose tolerance test, HOMA-IR - Homeostatic Model Assessment of Insulin } \\
\text { Resistance }\end{array}$} \\
\hline
\end{tabular}

to their counterparts [1]. It has been reported that the incidence of metabolic syndrome in adults increases with higher SUA concentration [1]. Hyperuricaemia has been described as a causative factor of vascular changes leading to hypertension in rats. This effect seems to be reversible in the early stages, but leads to unchangeable abnormalities in vessels walls. Hypotensive effect of uric acid lowering agents shows that this mechanism may occur in humans as well. SUA has been described as a marker for hypertension in both paediatric and adult populations [2].

Human immunodeficiency virus (HIV) infection itself, as well as antiretroviral treatment, are factors promoting metabolic abnormalities leading to cardiovascular disease. It has been widely described in both adult and paediatric populations. Children are of special concern due to lifespan therapy. Children infected with HIV are characterised by hyperlipidaemia, insulin resistance, and altered body fat accumulation; however, the prevalence of metabolic syndrome is low, mainly due to observed growth retardation. Cardiovascular risk, assessed by measurement of carotid intima-media thickness (cIMT), is also heightened; however, cardiovascular episodes are not observed in this population. It is necessary to establish an easy path for the assessment of cardiovascular risk, especially in resource-limited settings, thus the level of SUA may serve as a surrogate marker for cardiovascular disease (CVD).

The aim of the study was to assess SUA concentration and its association with risk factors for cardiovascular disease in HIV-infected children treated with highly active antiretroviral therapy (HAART).

\section{Material and methods}

A cross-sectional study was carried out in HIV-infected children. We included 54 children under care of the Department of Children's Infectious Disease Medical University of Warsaw (Table 1). Our centre takes care of approximately $70 \%$ of children infected with HIV in Poland. Legal guardians and patients, if aged above 16 years, signed a written informed consent. The study protocol was approved by Ethics Committee of the Medical University of Warsaw.

Every subject was examined during standard hospitalisation as part of routine care. None had acute infection, and none received steroids in the three months before the visit. All but two were treated with combination antiretroviral therapy (cART), both due to not fulfilling the recommendations to implement treatment: one after newly diagnosed HIV infection, the second after his own decision to stop therapy. We collected data about treatment regimen, nadir, and current immunological and clinical classification according to Centers for Disease Control and Prevention (CDC).

\section{Anthropometric measurements}

Every child underwent measurement of height and weight. Height was measured to the nearest $0.5 \mathrm{~cm}$, weight to the nearest $0.1 \mathrm{~kg}$. Body mass index (BMI) was calculated as weight $(\mathrm{kg}) /$ height $(\mathrm{m})^{2}$. BMI was expressed as $z$-score, using the formula:

BMI $z$-score $=(((\mathrm{XM}) \mathrm{L})-1) / \mathrm{LS}$,

where $X$ is the obtained BMI value, $L$ is the power in the Box-Cox transformation, $M$ is the median, and $S$ is the generalised coefficient of variation. Children with $z$-score values between $85^{\text {th }}$ and $95^{\text {th }}$ percentile were classified as overweight, and when $z$-score was above the $95^{\text {th }}$ percentile the child was classified as obese.

Blood pressure (BP) measurement was performed using a sphygmomanometer with a cuff size adjusted to the arm circumference. Results was expressed as a $z$-score. BP $z$-score was calculated using the formula:

BP $z$-score $=(\mathrm{x}-\mu) / \sigma$,

where $x$ is the obtained BP value, $\mu$ is the expected value of BP for age, and $\sigma$ is the standard deviation [6]. Children with BP $z$-score below 1.28 ( $90^{\text {th }}$ percentile) were classified as a normotensive, with $z$-score value between $1.28\left(90^{\text {th }}\right.$ percentile) and 1.645 ( $95^{\text {th }}$ percentile) were classified as pre- 
hypertensive, and with $z$-score exceeding 1.645 were classified as hypertensive.

Blood samples to all laboratory tests (SUA, insulin and glucose concentration, lipids) were obtained after a night fasting rest, then an oral glucose tolerance test was performed by administering a glucose in a dose of $1.75 \mathrm{~g}$ per kilogram body mass, max. $75 \mathrm{~g}$. Blood samples were collected twice during the oral glucose tolerance test (OGTT): 60 and 120 minutes after glucose administration. Homeostasis model assessment for insulin resistance was used to estimate insulin resistance. It was calculated using the formula:

HOMA-IR $=($ insulin $[\mathrm{mU} / \mathrm{l}] \times$ glucose $[\mathrm{mmol} / \mathrm{l}]) / 22.5$.

Insulin resistance was defined as HOMA-IR values exceeding 3.16 [7] (Table 2).

Received results of cholesterol and its fractions were multiple by 38.67 and triglycerides by 88.57 , to obtain values in $\mathrm{mg} / \mathrm{dl}$. Hypercholesterolaemia was diagnosed with values $>200 \mathrm{mg} / \mathrm{dl}$, for hypertriglyceridaemia $>100$ when aged $<9$ years, $>130 \mathrm{mg} / \mathrm{dl}$ for older than 9 years, hypo-HDL-C $<40$, and hyper-LDL-C $>130[8,9]$.

\section{Statistical methods}

Statistical calculations were performed using Statistica 13 (StatSoft). Continuous data were tested with Shapiro Wilk test to establish normal and non-normal distribution, and were shown as mean with SD. The continuous data were compared using $t$-test or $U$ Mann-Whitney test depending on distribution.

Two linear regression analyses were performed to identify variables associated with SUA concentration. Variables included in the first model were age, sex, BMI $z$-score, systolic blood pressure (SBP) $z$-score, diastolic blood pressure (DBP) $z$-score, and waist circumference above $90 \mathrm{c}$. Variables in the second model included total cholesterol (TC), triglyceride (TG), high-density lipoprotein cholesterol (HDL-C), low-density lipoprotein cholesterol (LDL-C), non-HDL-C, insulin, and HOMA-IR.

Statistical significance was set at the level $p<0.05$.

\section{Results}

The study group consisted of 54 children treated in the Department of Children's Infectious Diseases of the Medical University of Warsaw, aged 4-19 years (mean 12.3), 25 (46\%) boys. All children but two received HAART. In the study group none had severe immunodeficiency, four (7\%) had moderate immunodeficiency, and 50 (93\%) had no immunodeficiency, based on CD4 count. Forty-seven patients (87\%) showed no or mild clinical symptoms (group N or A by CDC), one presented group B clinical symptoms by $\mathrm{CDC}$, and six were classified as group C. All six children were classified to one immunological category. Clinical category $\mathrm{C}$ was due to HIV encephalopathy diagnosis. The worst clinical classification N/A, B, and C was in 24 (44.4\%), 10 (18.5\%), and $19(35.1 \%)$ patients, respectively. Sixteen (29.6\%)
Table 2. Correlation of serum uric acid (SUA) and metabolic parameters

\begin{tabular}{l|c|c}
\hline \multirow{2}{*}{ Factor } & \multicolumn{2}{|c}{ SUA } \\
\cline { 2 - 3 } & $r$ & $p$ \\
\hline Age & 0.4352 & 0.001 \\
\hline BMI z-score & 0.1039 & 0.455 \\
\hline SBP z-score & -0.0007 & 0.996 \\
\hline DBP z-score & -0.073 & 0.6 \\
\hline TC & -0.1666 & 0.233 \\
\hline TG & 0.3959 & 0.004 \\
\hline HDL & -0.3578 & 0.01 \\
\hline LDL & 0.0748 & 0.598 \\
\hline Insulin & 0.3787 & 0.005 \\
\hline Fasting glucose & 0.2185 & 0.12 \\
\hline Glucose in 120' OGTT & 0.2213 & 0.122 \\
\hline HOMA-IR & 0.3193 & 0.021 \\
\hline BM-body mass index, SBP-systolc blod & & \\
\hline
\end{tabular}

$B M I$ - body mass index, SBP - systolic blood pressure, DBP - diastolic blood pressure, TC - total cholesterol, TG - triglyceride, HDL - high-density lipoprotein, $L D L$ - low-density lipoprotein, OGTT - oral glucose tolerance test, HOMA-IR - Homeostatic Model Assessment of Insulin Resistance

Table 3. Comparison of serum uric acid (SUA) between groups with and without metabolic abnormalities

\begin{tabular}{|c|c|c|c|}
\hline \multicolumn{2}{|l|}{ Factor } & SUA $(\mu \mathrm{mol} / \mathrm{l})$ & $p$ \\
\hline \multirow{2}{*}{ Hypertension } & No & 239.70 & \multirow{2}{*}{0.940} \\
\hline & Yes & 240.20 & \\
\hline \multirow{2}{*}{ Hyper-TC } & No & 239.89 & \multirow{2}{*}{0.888} \\
\hline & Yes & 229.33 & \\
\hline \multirow{2}{*}{ Hyper-TG } & No & 209.15 & \multirow{2}{*}{0.012} \\
\hline & Yes & 254.16 & \\
\hline \multirow{2}{*}{ Hypo-HDL-C } & No & 232.00 & \multirow{2}{*}{0.055} \\
\hline & Yes & 284.83 & \\
\hline \multirow{2}{*}{ Hyper-LDL-C } & No & 237.89 & \multirow{2}{*}{0.989} \\
\hline & Yes & 228.83 & \\
\hline \multirow{2}{*}{ IR } & No & 222.41 & \multirow{2}{*}{0.015} \\
\hline & Yes & 266.78 & \\
\hline \multicolumn{4}{|l|}{ HAART scheme } \\
\hline \multirow{2}{*}{$2 \mathrm{NRTI}+\mathrm{NNRTI}$} & Yes & 193.68 & \multirow{2}{*}{0.000003} \\
\hline & No & 264.80 & \\
\hline \multirow{2}{*}{$2 \mathrm{NRTI}+\mathrm{PI}$} & yes & 265.55 & \multirow{2}{*}{0.012} \\
\hline & No & 222.06 & \\
\hline \multirow{2}{*}{ Other } & yes & 275.27 & \multirow{2}{*}{0.020} \\
\hline & No & 230.70 & \\
\hline
\end{tabular}

HAART - highly active antiretroviral therapy, NRTI - nucleoside reverse transcriptase inhibitor, NNRTI - non-nucleoside reverse transcriptase inhibitor, $\mathrm{Pl}$ - protease inhibitor 
patients were in group 1 immunological nadir classification, $20(37 \%)$ in group 2, and $17(31.4 \%)$ in group 3. We lacked data on nadir clinical and immunological classification in one patient. Mean concentration of SUA was $239.8 \mu \mathrm{mol} / \mathrm{l}$, no subject had a level exceeding the normal range. Boys had significantly higher levels of SUA. Higher levels of SUA were associated with age, higher level of triglycerides, insulin, and HOMA-IR, and lower levels of HDL-C. The correlation between SUA and metabolic parameters is shown in Table 3.

Analysis of the impact of advancement of clinical and immunological disorders on SUA showed that children with nadir severe immunodeficiency (stage 3 according to CDC classification) had significantly higher SUA.

SUA level comparison between groups with and without hypertension, hypercholesterolaemia, hypertriglyceridaemia, hypo-HDL-cholesterolaemia, hyper-LDL-cholesterolaemia, and insulin resistance showed significant differences only in relation to hyper-TG and IR. Children with hypo-HDL-cholesterolaemia had higher values of SUA, but the differences did not reach statistical significance $(p=0.055)$.

Analysis of factors that may impact SUA levels, including HAART agents, showed strong association with the ad-

Table 4. Multivariate regression model of the anthropometric predictors of serum uric acid (SUA) level

\begin{tabular}{l|c|c}
\hline Factor & $\boldsymbol{\beta}$ & $\boldsymbol{p}$ \\
\hline Age at assessment & 0.41 & 0.003 \\
\hline Gender male & -0.32 & 0.01 \\
\hline BMI z-score & 0.01 & 0.12 \\
\hline SBP z-score & 0.07 & 0.62 \\
\hline DBP z-score & 0.02 & 0.88 \\
\hline Waist circumference $>90 \mathrm{C}$ & 0.01 & 0.92 \\
\hline $\mathrm{R}^{2}$ of the model & 0.3 & 0.007 \\
\hline
\end{tabular}

$B M I$ - body mass index, SBP - systolic blood pressure, DBP - diastolic blood pressure

Table 5. Multivariate regression model of the metabolic predictors of serum uric acid (SUA) level

\begin{tabular}{l|c|c}
\hline Factor & $\boldsymbol{\beta}$ & $\boldsymbol{p}$ \\
\hline TC & 0.43 & 0.02 \\
\hline TG & 0.54 & 0.004 \\
\hline HDL-C & 0.27 & 0.24 \\
\hline LDL-C & 0.78 & 0.03 \\
\hline Insulin & -1.59 & 0.009 \\
\hline HOMA-IR & 1.76 & 0.003 \\
\hline$R^{2}$ of the model & 0.44 & 0.0004
\end{tabular}

TC - total cholesterol, TG - triglyceride, HDL-C - high-density lipoprotein cholesterol, LDL-C - low-density lipoprotein cholesterol, HOMA-IR - Homeostatic Model Assessment of Insulin Resistance ministered treatment. Children treated with non-nucleoside reverse transcriptase inhibitors (NNRTIs)-based HAART showed significantly lower SUA concentrations, unlike the results of either protease inhibitor (PI)-based or other HAART schemes.

The associations between SUA concentration and anthropometric and metabolic parameters are shown in Tables 4 and 5. Among anthropometric confounders age and sex significantly influenced SUA concentration. The metabolic parameters model showed association between SUA and each confounder except HDL-C.

\section{Discussion}

In this cross-sectional study among HIV infected children and adolescents we found an association between SUA and traditional markers for CVD such as TC, TG, low-density lipoprotein (LDL), high-density lipoprotein (HDL), or insulin resistance. PI exposure was associated with higher levels of SUA in opposition to NNRTI exposure. Uric acid has been recently described in the general paediatric population as a marker of risk factor for future cardiovascular disease. The paediatric population is of special concern because atherosclerosis begins early in childhood and remains asymptomatic for a long time. Therefore, it is necessary to assess markers that give the possibility to recognise children at risk as early as possible, to be able to stop progress of the disease and to avoid developing clinical symptoms.

Hyperuricaemia plays an important role in developing hypertension, as well as in type II diabetes, and remains an independent risk factor for cardiovascular disease. Results of the Framingham study showed increased risk of ischaemic heart disease or cardiac arrest in patients with high concentrations of SUA. The Polish Society of Hypertension recommends consideration of implementation of allopurinol in patients with hypertension and asymptomatic hyperuricaemia $[10,11]$.

The biologic mechanism of uric acid in promoting hypertension seems to be two-stage: the first is reversible, unlike the second. The first phase is the result of increased renin production and decreased circulating plasma nitrates, which leads to excessive vasoconstriction. After lowering the SUA concentration the blood pressure decreases proportionally to SUA reduction. Long exposure to high SUA levels leads to rebuilt in renal vascular. Reaching of smooth muscle cells by uric acid anion transporter 1 (UAAT-1) results in its proliferation mediated by PDGF and MCP-1. The ultimate effect is reduced compliance of renal afferent arterioles, shifted pressure natriuresis curve, and sodium-sensitive hypertension. This level cannot be modified by SUA lowering agents [12-15]. A hypertensive effect of uric acid has been described in rats; however, it seems to be transferable to humans. Moreover, it has been shown that uric acid lowering agents have a hypotensive effect, regardless of the drug mechanism [16]. It may be a modifiable risk factor for hypertension, so study results support a favourable effect of lowering SUA [16]. On the other hand, it has been reported that higher levels 
of uric acid limits changing lifestyle and body mass reduction effects on BP values. It is of special concern that uric acid, independently of other risk factors (i.e. insulin resistance or overweight/obesity), affects future hypertension [17]. Therefore, it is possible that uric acid promotes hypertension regardless of other known risk factors, and its lowering may be beneficial as the only intervention.

SUA was initially associated with essential hypertension as well as with prehypertension, especially in the overweight/obese $[12,18]$. The predictive value of increased uric acid is not limited to hypertension, but it refers also to cardiovascular disease, obesity, and metabolic syndrome. A relationship between insulin resistance and hypertension has been reported in both adults and children. Insulin resistance is a predictor for hypertension independently of traditional risk factors, e.g. obesity [19]. This shows that insulin resistance promotes the development of hypertension not only through impaired metabolism of fat tissue but it also has its own pattern. Our study results showed an association between SUA and both HOMA-IR level and insulin resistance.

HIV infection has been widely described as a risk factor for metabolic abnormalities and early atherosclerosis. Both HIV itself and HAART promote the abnormalities independently. It remains clinically invisible for a long time; however, the lifespan exposure to infection and treatment cause cardiovascular episodes earlier than in the healthy population.

Nowadays, due to HAART, HIV-infected children are expected to live to adulthood. The incidence of the acquired immunodeficiency syndrome (AIDS) condition is decreasing, and it is replaced by non-AIDS defining diseases - mostly side effects of HAART, among which metabolic abnormalities are prevalent. Dyslipidaemia and insulin resistance are widely described in HIV-infected children and adolescents, with prevalence differing between studies. One of the reasons for this is a multifactorial condition with causes surpassing of HIV infection and HAART. These are related to lifestyle, diet, physical activity, and heredity. Results of studies assessing the influence of HAART on metabolic abnormalities are consistent. PI exposure is associated with increased prevalence and advancement of lipid and glucose metabolism abnormalities, whereas NNRTI shows the opposite influence. The beneficial effect of INSTI on lipid profile seen in adults may occur also in children; however, the drugs are registered in patients above 12 years old [20]. Children and youths are of special concern mainly due to a life exposure to HIV and HAART. However, it is particularly important to recognise and establish reliable markers for future cardiovascular disease.

There have been several studies assessing the incidence of hypertension in HIV-infected children. The results remain inconsistent. Our study group was characterised by similar incidence of increased blood pressure as in data from other studies. Surprisingly, subjects with and without hypertension had similar SUA levels. It is uncommon regarding data from studies describing SUA as a marker for hypertension and prehypertension. It has also been assessed as marker for future hypertension, with excellent correlation with risk factors, e.g. obesity or heredity.

Data on the prevalence of hypertension in HIV-infected children are inconsistent. Our small population showed a hypertension prevalence of $16 \%$, which is higher than the prevalence reported by Sainz et al. [21] and more similar to results of Chatterton-Kirchmeier et al. [22]. We did not perform a comparison with uninfected children; however, our prevalence is much higher than the approximately $4 \%$ estimated in the Polish population [23]. Data from most studies show no differences in both prevalence of hypertension and values of blood pressure between HIV-infected and uninfected children [21,24-26]. No association between SUA and blood pressure or hypertension was shown. It is surprising due to multiple study results showing a strong correlation between blood pressure and SUA.

Risk factors for hypertension seem to be similar to those for other metabolic abnormalities in HIV infection. It is the result of multiple overlapping factors: HIV itself, persistent immunological activation, and HAART side effects. We are not able to accurately identify the border between them, so the results of studies assessing risk factors are difficult to interpret and transfer to clinical settings. Metabolic abnormalities are better recognised mainly as side effects of HAART. Until now only a few studies have assessed hypertension in HIV-infected children, but none have reported an association between HT and HAART. Our results show correlation with not all metabolic parameters but with those especially connected with antiretrovirals. Moreover, strong correlation between SUA and HAART, with typical pattern, e.g. cardioprotective activity of NNRTI and opposite result of PI, indicates that factors resulting from HIV infection may play a leading role in developing hypertension in this specific population. However, the question of whether SUA is part of the pathological process leading to CVD or a result of the process remains unanswered. Lack of correlation between hypertension and SUA sustain that HIV and associated factors may outweigh traditional risk factors.

\section{Conflict of interest}

The authors declare no potential conflicts of interest with respect to the research, authorship, and/or publication of this article.

\section{References}

1. Valle M, Martos R, Canete MD, et al. Association of serum uric acid levels to inflammation biomarkers and endothelial dysfunction in obese prepubertal children. Pediatr Diabetes 2015; 16: 441-447.

2. Cardoso AS, Gonzaga NC, Medeiros CC, de Carvalho DF. Association of uric acid levels with components of metabolic syndrome and non-alcoholic fatty liver disease in overweight or obese children and adolescents. J Pediatr (Rio J) 2013; 89: 412-418.

3. Bassols J, Martínez-Calcerrada J, Prats-Puig A, et al. Uric acid, carotid intima-media thickness and body composition in prepubertal children. Pediatr Obes 2015; 11: 375-382. 
4. Ford ES, Li C, Cook S, Choi HK. Serum concentrations of uric acid and the metabolic syndrome among US children and adolescents. Circulation 2007; 115: 2526-2532.

5. Manco M, Nobili V, Alisi A, et al. Arterial stiffness, thickness and association to suitable novel markers of risk at the origin of cardiovascular disease in obese children. Int J Med Sci 2017; 14: 711-720.

6. National High Blood Pressure Education Program Working Group on High Blood Pressure in Children and Adolescents. The fourth report on the diagnosis, evaluation, and treatment of high blood pressure in children and adolescents. Pediatrics 2004; 114 (2 Suppl $4^{\text {th }}$ Report): 555-576.

7. Keskin M, Kurtoglu S, Kendirci M, et al. Homeostasis model assessment is more reliable than the fasting glucose/insulin ratio and quantitative insulin sensitivity check index for assessing insulin resistance among obese children and adolescents. Pediatrics 2005; 115: e500-503.

8. Expert Panel on Integrated Guidelines for Cardiovascular Health and Risk Reduction in Children and Adolescents: Summary Report. Pediatrics 2011; 128 (Suppl 5): S213-S256.

9. McBride PE, Kavey RE. Lipid screening and treatment recommendations for children and adolescents. Pediatr Ann 2012; 41: 1-10.

10. Widecka K, Szymański FM, Filipiak KJ, et al. Stanowisko ekspertów dotyczące hiperurykemii i jej leczenia u pacjentów z wysokim ryzykiem sercowo-naczyniowym. Arter Hypertens 2017; 21: 1-9.

11. Tykarski A, Narkiewicz K, Gaciong Z, et al. 2015 Guidelines for the Management of Hypertension. Arter. 2015; 19: 53-83. doi: 10.1016/j.mcna.2016.08.016.

12. Wang Y, Hu JW, Lv YB, et al. The role of uric acid in hypertension of adolescents, prehypertension and salt sensitivity of blood pressure. Med Sci Monit 2017; 23: 790-795.

13. Yanik M, Feig DI. Serum urate: a biomarker or treatment target in pediatric hypertension. Curr Opin Cardiol 2013; 28: 433-438.

14. Feig DI, Madero M, Jalal DI, et al. Uric acid and the origins of hypertension. J Pediatr 2012; 162: 896-902.

15. Feig DI, Johnson RJ. The role of uric acid in pediatric hypertension. J Ren Nutr 2007; 17: 79-83.

16. Soletsky B, Feig DI. Uric acid reduction rectifies prehypertension in obese adolescents. Hypertension 2012; 60: 1148-1156.

17. Viazzi F, Rebora P, Giussani M, et al. Increased serum uric acid levels blunt the antihypertensive efficacy of lifestyle modifications in children at cardiovascular risk. Hypertension 2016; 67: 934-940.

18. Viazzi AF, Antolini L, Giussani M, et al. Serum uric acid and blood pressure in children at cardiovascular risk. Pediatrics 2013; 132 : e93-e99.

19. Genovesi S, Brambilla P, Giussani M, et al. Insulin resistance, prehypertension, hypertension and blood pressure values in paediatric age. J Hypertens 2012; 30: 327-335.

20. Gatell JM, Assoumou L, Moyle G, et al. Switching from a ritonavir-boosted protease inhibitor to a dolutegravir-based regimen for maintenance of HIV viral suppression in patients with high cardiovascular risk. AIDS 2017; 31: 2503-2514.

21. Sainz T, Alvarez-Fuente M, Navarro ML, et al. Subclinical atherosclerosis and markers of immune activation in HIV-infected children and adolescents: The CaroVIH Study. J Acquir Immune Defic Syndr 2014; 65: 42-49.

22. Chatterton-Kirchmeier S, Camacho-Gonzalez AF, Mccracken CE, et al. Increased prevalence of elevated blood pressures in HIV-infected children, adolescents and young adults. Pediatr Infect Dis J 2015; 34: 610-614.

23. Żurowska A, Zwolińska D, Roszkowska-Blaim M, et al. Rekomendacje Polskiego Towarzystwa Nefrologii Dziecięcej (PTNFD) dotyczące postępowania $\mathrm{z}$ dzieckiem $\mathrm{z}$ podwyższonym ciśnieniem tętniczym. Forum Med Rodz 2015; 9: 349-375.

24. Idris NS, Grobbee DE, Burgner D, et al. Effects of paediatric HIV infection on childhood vasculature. Eur Heart J 2016; 37: 3610 3616.
25. Sainz T, Diaz L, Navarro ML, et al. Cardiovascular biomarkers in vertically HIV-infected children without metabolic abnormalities. Atherosclerosis 2014; 233: 410-414.

26. Giuliano IDCB, de Freitas SFT, de Souza M, Caramelli B. Subclinic atherosclerosis and cardiovascular risk factors in HIV-infected children: PERI study. Coron Artery Dis 2008; 19: 167-172. 